\title{
Navigating in the labyrinth of thrombotic and bleeding risks in patients with malignancies - how to make the most reasonable choices for personalized anticoagulation?
}

\author{
Katarzyna (Kate) Rygiel ${ }^{1}$
}

Cite this article: Rygiel K: Navigating in the labyrinth of thrombotic and bleeding risks in patients with malignancies - how to make the most reasonable choices for personalized anticoagulation? AsiaPac J Oncol 2020; 1: 68-76. https://doi. org/10.32948/ajo.2020.12.31

\begin{abstract}
Venous thromboembolism (VTE) frequently occurs among patients with malignancies and poses an important cause of morbidity and mortality in this population. Therefore, effective and safe thromboprophylaxis for oncology patients at the increased risk of VTE is of utmost importance. Commonly used anticancer treatments, including hormonal therapy $(\mathrm{HT})$, chemotherapy ( $\mathrm{CHT}$ ), targeted therapy (TT), immune therapy (IT), radiotherapy (RT), and anti-angiogenesis monoclonal antibodies, as well as surgical procedures have been associated with VTE. For this reason, risk stratification scores, including tumor site, laboratory parameters, and patient's clinical characteristics can help most accurately identify those patients, who will take the greatest advantage of a personalized approach to VTE.

This mini-review discusses cancer-related VTE risk stratification scores (e.g., the Khorana, Vienna Cancer and Thrombosis (CATS), and PROTECHT) that have been very useful for the detection of patients at the highest risk of VTE, who require an individual choice of the anticoagulant. This article briefly summarizes the updated American Society of Clinical Oncology (ASCO) clinical guidelines for the prevention and treatment of VTE in patients with cancer. In particular, it presents the direct oral anticoagulants (DOACs) as a new opportunity for both the preventive and therapeutic approach to VTE in this population. Furthermore, this overview provides some practical implications of the ASCO recommendations to the decisionmaking regarding safe and effective, personalized anticoagulant selection in various clinical setting. Hopefully, blending the patient's medical context and personal preferences into VTE risk stratification scores will contribute to progress in the management of cancer-related VTE.
\end{abstract}

Key words Venous thromboembolism (VTE), non-vitamin $\mathrm{K}$ antagonist oral anticoagulants (NOACs), direct oral anticoagulants (DOACs), VTE risk stratification scores, Khorana Score (KS), cancer-related VTE

1. Department of Family Practice, Medical University of Silesia (SUM), Zabrze, Poland.

Correspondence: Katarzyna (Kate) Rygiel (Department of Family Practice, Medical University of Silesia (SUM), 3 Maja St, 41-800 Zabrze, Poland ; E-mail: kasiaalpha@yahoo.co.uk). 


\section{Introduction}

Thrombotic complications, such as venous thromboembolism (VTE), frequently occur among patients with malignancies, contributing to their increased morbidity and death [1]. The increased VTE risk, as well as the bleeding risk, have been highest in the first year, after the initial diagnosis of neoplastic disease [1]. It should be emphasized that patients with cancer who have experienced recurrent episodes of VTE have a four-times higher mortality risk compared to the ones without VTE [2].

Cancer-related hypercoagulability includes type I (caused by the enzymatic degradation of endogenous heparin by heparanase from a tumor) and type II (relevant to factors linked to the patient, the tumor, and its therapy) [3]. Moreover, patients with malignancies, who are anticoagulated, have a higher incidence of bleeding compared with patients without cancer, regardless of the selected anticoagulation agent [4]. In addition, the patients, who suffer from metastatic disease, gastrointestinal (GI), gynecological or genitourinary (GU) cancers, thrombocytopenia, coagulopathy, and major bleeding episode, are usually characterized by higher bleeding risk [4].

Under these circumstances, clinical cancer-related VTE risk stratification scores have been useful to detect oncology patients with the greatest risk of VTE (Figure 1) [5-7]. Such risk scores include the primary anatomic site and histologic type of cancer, complete blood count (CBC) before administration of chemotherapy (CHT) (e.g., hemoglobin level, white blood cells, and platelets), body mass index (BMI), and soluble biomarkers (e.g., D-dimer and P-selectin) (Figure 1) [5-7].

This mini-review discusses the role of cancer-related VTE risk stratification scores (e.g., the Khorana, Vienna Cancer and Thrombosis (CATS), and PROTECHT) that have been very helpful (according to the results of the main randomized controlled trials (RCTs) focused on cancer-related VTE) for the identification of patients with the highest risk of VTE, who require an individual choice of the most optimal anticoagulant. Furthermore, it briefly summarizes the updated American Society of Clinical Oncology (ASCO) clinical guidelines for the management of VTE among patients with cancer. In particular, it presents the direct oral anticoagulants (DOACs), such as apixaban, edoxaban, and rivaroxaban, as a new treatment opportunity for both the prevention and treatment of VTE in patients with cancer. This overview also provides some practical implications of the ASCO recommendations to the decision-making regarding safe and effective, individualized anticoagulant choices in the oncology practice setting. Hopefully, blending the patient clinical context and personal preferences into VTE risk stratification scores will contribute to some progress in therapy and prevention of cancerrelated VTE.

\section{Understanding the main mechanisms contributing to hypercoagulability in patients with malignancies}

Among patients with malignancies, different mechanisms can affect their pro-thrombotic risk. The association between malignant diseases and thrombotic events has been known for many decades. However, the exact underlying mechanisms are still not completely elucidated. Endogenous heparin is necessary to achieve an equilibrium between circulating blood fluidity and the coagulation process. However, in many patients with cancerrelated VTE, the enzymatic degradation of endogenous heparin by heparanase contributes to cancer-associated VTE, together with a plethora of other factors, linked to cancer itself, its treatment, and an individual patient's clinical and personal profile. In fact, stasis (due to pressure of the tumor on venous or arterial vessel walls) results in hemodynamic dysfunctions, as well as changes in the constituents of the blood, which can contribute to thrombosis [8]. Moreover, several systemic anticancer therapies are correlated with VTE or arterial thrombosis. Surgical interventions, often followed by bed-rest or inactivity, are also related to an augmented risk of VTE. Procoagulant molecules secreted from tumor cells are the main cause of hypercoagulability in cancer patients. Heparanase is a mammalian endoglycosidase that degrades heparan sulfate at the cell surface and in the extracellular matrix. Heparanase is physiologically expressed in platelets and the placenta and is pathologically overexpressed in most malignant tumors. Importantly, low-molecular-weight heparin (LMWH) is a competitive inhibitor of heparanase [9].

\section{Two types of cancer-related hypercoagulability and their} clinical implications

In general, a hypercoagulable state among patients with cancers has been categorized as type I (which occurs when the proportion between the endogenous heparin production and its degradation is altered, via accelerated degradation of endogenous heparin by an enzyme heparanase, secreted by a tumor), and type II (which involves other factors relevant to the malignant tumor itself, its treatment, and to the patient clinical or personal profile) [3].

Type I cancer-related hypercoagulability is characterized by recurrent VTE episodes, in patients with malignancies, due to an insufficient amount of the endogenous heparin, to keep the blood in its natural liquid state. This is predominantly caused by a degradation of the endogenous heparin by heparanase (an enzyme endoglycosidase), secreted by a tumor [10]. In fact, pancreatic cancers are characterized by the heparanase mRNA levels, which are above 30-times higher, compared to the ones encountered physiologically in the pancreatic gland [11]. Moreover, it has been shown that overexpression of heparanase was associated with poor prognosis in a majority of patients with lung cancer [12]. Similarly, a large meta-analysis of patients with gastric cancer has revealed that higher heparanase expression in gastric cancer was related to the tumor invasiveness, metastases to lymph nodes, and TNM stage [13]. Furthermore, some tumors can contribute to the narrowing of blood vessel's lumen and slowing down local circulation, which in turn generates abnormal hemodynamic forces leading to endothelial dysfunction that often results in VTE [14].

In addition, the tissue factor (TF) represents a common signal for coagulation, and in particular, the TF upregulation in some cancers may lead to a hypercoagulable state [15]. Similarly, the upregulation of lysyl oxidase (LOX), which is an enzyme responsible for the cross-linking of collagen, and for the increase of platelet's reactivity, can also elevate the VTE risk in many patients with neoplastic diseases [16]. Overexpression of heparanase and its excessive secretion by cancer cells often leads to a degradation of endogenous heparin and increased coagulability. In addition, heparanase can induce TF expression in vascular endothelium and neoplastic cells [17].

Type II cancer-related hypercoagulability predominantly relates to VTE events, which are not connected with the decreased levels of endogenous heparin. Type II predominantly involves a combination of stasis (secondary to pressure exerted on blood vessels by a tumor mass), the patient's poor performance status, obesity, and anti-neoplastic therapy-related thrombosis (Figure 2) [18]. In addition, numerous anticancer therapies, such as hormonal therapy (HT), chemotherapy (CHT), targeted therapy (TT), immune therapy (IT), radiotherapy (RT), and anti-angiogenesis monoclonal antibodies are related to an elevated risk of VTE. Moreover, many patients who undergo surgery, often associated with the insertion of central venous catheters (or other devices), are immobilized or inactive, and thus, have a higher risk of VTE. 


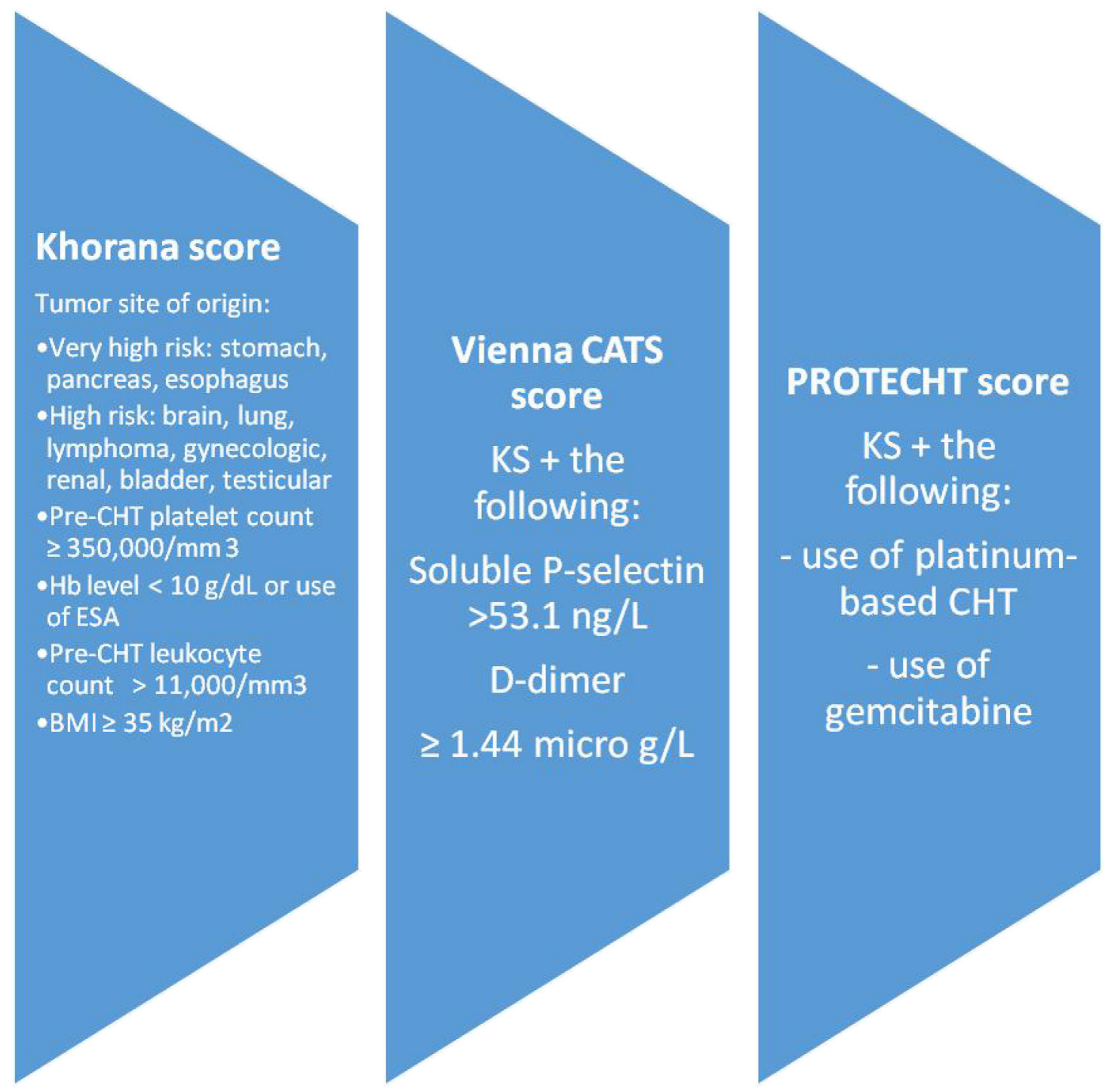

Figure 1. Risk stratification scores (Khorana, Vienna Cancer and Thrombosis (CATS), PROTECHT) for VTE risk assessment in patients with cancer [5, 6, 7]. Abbreviations: BMI, body mass index; CHT, chemotherapy; ESA, erythropoietin stimulating agent; Hb, hemoglobin; VTE, venous thromboembolism.

(Figure 2) [18].

The advantages of clinical prediction scores in patient's selection for thromboprophylaxis of cancer-related VTE

At present, guidelines from the American Society of Clinical Oncology (ASCO) recommend primary prophylaxis of VTE (e.g., prior to VTE event) for in-patients, who receive therapy for their active cancers, and for those with multiple myeloma, on immunomodulatory treatment (Table 1) [19]. Furthermore, thromboprophylaxis should be considered for certain high-risk patients, in whom a tumor site of origin is related to the highest risk of VTE, such as pancreatic ductal adenocarcinoma and gastric adenocarcinoma [20]. In addition, cancer stage is related to the risk of VTE, and in fact, many patients with metastases have a twentytimes increased risk of VTE, compared to those without cancer.
[21]. Similarly, biomarkers including TF, D-dimer, and P-selectin have also been revealed as helpful predictors for an increased risk of cancer-related VTE [22, 23].

Numerous clinical prediction scores have been created to best identify those patients who may need primary VTE prophylaxis, due to their individual risk profiles (Figure 1) [5]. The Khorana score was the first, well-validated clinical tool to predict VTE risk, specifically in patients with cancer [24]. Advantages of the Khorana scoring include its simplicity, and high negative predictive value, allowing physicians to exclude low-risk patients from thromboprophylaxis and related bleeding risks [5]. Limitations of the Khorana scoring involve a low positive predictive value, a need for further risk stratification (since many patients are classified as an intermediate risk), and no consistent validity in single sites of cancers [5]. Other clinical prediction scores, including the Vienna score, and the Protecht score, which modify the original Khorana 


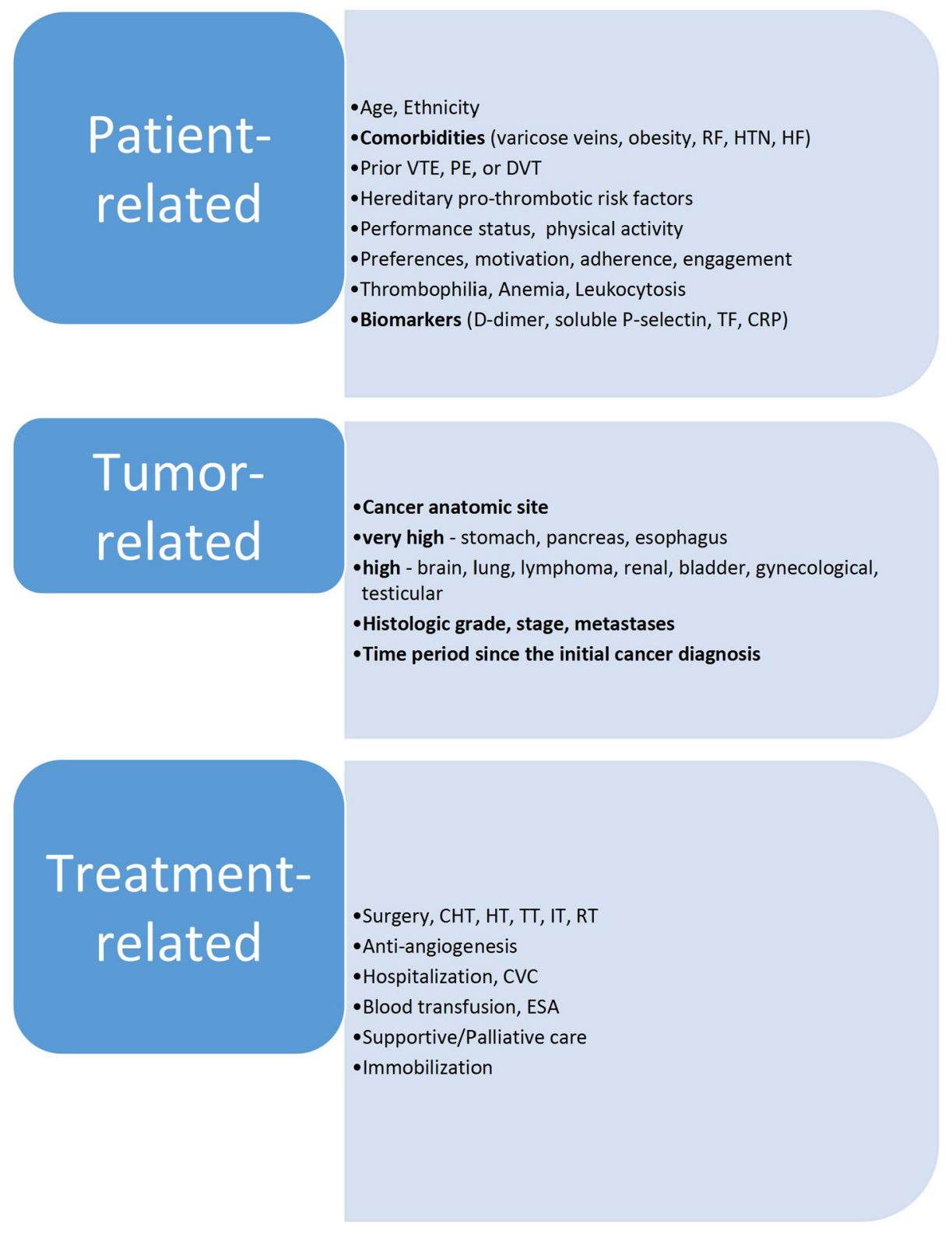

Figure 2. The main risk factors for thromboembolism in patients with cancer [18]. Abbreviations: CRP, C-reactive protein; DVT, deep venous thrombosis; ESA, erythropoietin stimulating agent; HF, heart failure; HTN, arterial hypertension; PE, pulmonary embolism; TF, tissue factor; VTE, venous thromboembolism; CHT, Chemotherapy; HT, Hormonal therapy; TT, Targeted therapy; IT, Immunotherapy; RT, Radiotherapy; CVC, central venous catheters; RF, renal failure; Hereditary pro-thrombotic risk factors (e.g., protein $\mathrm{C}$ deficiency, protein $\mathrm{S}$ deficiency, anti-thrombin deficiency, or factor $\mathrm{V}$ Leiden).

score, serve as useful clinical prediction instruments for VTE risk in patients with cancer (Figure 1) [6,7].

It should be noted that some randomized controlled trials (RCTs) have assessed the use of low molecular weight heparin (LMWH) for primary thromboprophylaxis in patients with cancer. In particular, semuloparin, an ultra- LMWH, was studied for efficacy and safety of thromboprophylaxis in a large group of unselected patients with locally advanced or metastatic cancer, receiving CHT [25]. After a median follow-up period of 3.5 months, VTE occurred in $1.2 \%$ of patients in the semuloparin group and in $3.4 \%$ of participants in the placebo group, and clinically relevant bleeding occurred in $2.8 \%$ of patients receiving semuloparin versus $2.0 \%$ of those receiving placebo [25]. When patients with cancer, at high risk of VTE (Khorana score of three or greater) were selected, thromboprophylaxis with dalteparin (LMWH) significantly reduced the VTE incidence (12\% in the LMWH group versus $21 \%$ in the observation group), but the LMWH use resulted in a seven-fold increase risk of bleeding [26]. Therefore, routine use of LMWH for thromboprophylaxis in cancer patients was not recommended for unselected patients, according to practice guidelines (except from patients with pancreatic cancer receiving CHT) [27].

A patient's poor performance status (e.g., according to the Eastern Cooperative Oncology Group $(\mathrm{ECOG}) \geq 2$ ) has been linked to an augmented risk of hypercoagulability. About $30 \%$ of patients with malignancies (e.g., in advanced stage), who were admitted to palliative care services suffered from a femoral deep vein thrombosis (DVT) [28]. Furthermore, surgical interventions, often associated with insertion of central venous catheters or other foreign devices, and immobilization, have increased the risk of 


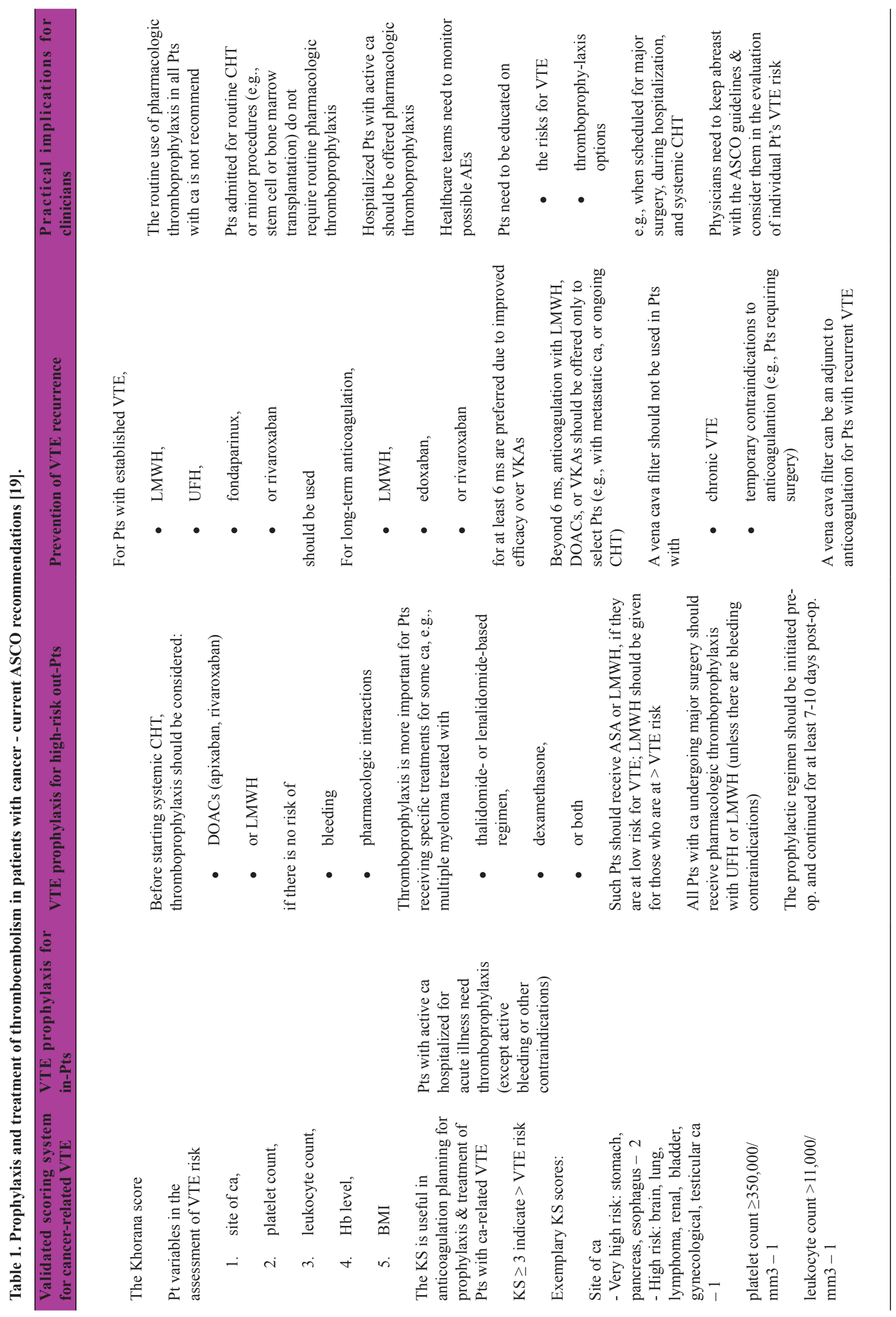


DVT or PE among patients with malignancies. In addition, elderly age, preexisting motor dysfunctions, diagnosis of brain tumor (e.g., high-grade glioma) or gastric cancer were related to higher risk of VTE or PE occurrence in pre- and post-operative period [29, 30].

\section{Correlations between CHT and increased incidence of VTE}

Multiple studies have shown correlations between CHT and increased incidence of VTE. For instance, a retrospective analysis involving 17,284 ambulatory patients with malignancies has shown that VTE occurred in $12.6 \%$ of the patients with different types of cancers, within one year after starting CHT, compared to only $1.4 \%$ of patients without cancers [31]. A commonly used CHT agent, cisplatin, has been related to an increased risk of both VTE and arterial thrombosis. A meta-analysis of RCTs assessing the risk of VTE related to cisplatin-based CHT has shown an elevated risk of VTE rates in patients treated with cisplatin-based vs. non-cisplatin-based CHT [32]. Moreover, a large RCT of patients with advanced gastroesophageal cancer, receiving epirubicin/(fluorouracil or capecitabine) and cisplatin or oxaliplatin, has reported fewer VTE events in the oxaliplatin arm, compared to the cisplatin arm ( $7.6 \%$ vs. $15.1 \%$, respectively), according to the UK National Cancer Research Institute [33]. Similarly, a retrospective U.S. analysis has revealed that $18.1 \%$ of patients, who developed VTE during therapy with cisplatin, had experienced thrombotic complications, mainly during the first three months from the beginning of their CHT course [34]. According to a RCT (conducted by the National Surgical Adjuvant Breast and Bowel Project (NSABP), B-14), which has compared the effectiveness of HT using tamoxifen in women with breast cancer (BC) (estrogen-receptor (ER) positive, with histologically negative axillary lymph nodes), VTE occurred in $0.9 \%$ of women in the tamoxifen arm, compared to only $0.15 \%$ in the placebo arm [35]. Moreover, VTE episodes were more frequently reported when CHT was administered in combination with tamoxifen, compared to tamoxifen used in monotherapy. The NSABP B-20 trial has compared CHT plus tamoxifen vs. tamoxifen as a monotherapy, in the treatment of women with ER-positive BC and negative axillary lymph nodes. VTE was noted in $1.8 \%$ of women treated with tamoxifen alone, compared to $6.5 \%$ in those, who were treated with tamoxifen, cyclophosphamide, methotrexate, and fluorouracil [36]. This elevated risk of VTE, when tamoxifen was used in combination with CHT, was the underlying reason behind the recommendation to withhold tamoxifen, until the CHT completion.

Similarly to many cytotoxic CHT agents, some targeted therapies reveal their pro-thrombotic properties in the oncology population. For instance, osimertinib (an epidermal growth factor receptor (EGFR) inhibitor, and lenvatinib (a tyrosine kinase receptor inhibitor (TKI), such as vascular endothelial growth factor (VEGF), fibroblast growth factor receptor, and plateletderived growth factor (PDGF) receptor alpha) can contribute to an increased risk of VTE and pulmonary embolism (PE) [37, 38]. Lenvatinib has been investigated (in combination with carboplatin and paclitaxel) in patients with non-small-cell lung cancer, and has shown thrombotic adverse effects [38]. Likewise, in a study exploring treatment of patients with advanced cancer of the thyroid gland, lenvatinib has been noted to cause complications, such as $\mathrm{PE}$ or DVT in $3 \%$ of the trial participants [39].

Bevacizumab is an anti-angiogenesis monoclonal antibody, which targets vascular endothelial growth factor (VEGF) in the circulating blood. The combination of bevacizumab with irinotecan, fluorouracil, and leucovorin has improved outcomes in patients with colorectal cancer, but VTE episodes were higher among patients treated with bevacizumab compared to those receiving CHT alone (19.4\% vs. $16.2 \%$, respectively) [40]. A meta- 
analysis of twenty RCTs has revealed that the incidence of arterial thrombosis in patients using bevacizumab was $3.3 \%$, and this risk was variable with different cancers (e.g., the highest relative risk of 3.72 was in patients with renal cell cancer, and the lowest of 1.89 , was in patients with colorectal cancer) [41].

Insights from the AVERT and CASSINI trials exploring the preventive role of DOACs in patients with cancer and elevated VTE risk

Two main RCTs, AVERT (Apixaban for the Prevention of Venous Thromboembolism in High-Risk Ambulatory Cancer Patients) and CASSINI (Rivaroxaban for Thromboprophylaxis in High-Risk Ambulatory Patients with Cancer), have explored the preventive role of DOACs in patients with cancer and elevated risk of VTE $[42,43]$. It should be underscored that the Khorana risk score was above 2 in the majority of participants of these RCTs.

In the AVERT trial, patients with an active malignancy receiving CHT (with a Khorana score of 2 or above) were randomized to apixaban (2.5 mg twice daily) or placebo for six months [42]. In the intention-to-treat (ITT) analysis, the apixaban group had a decreased incidence of VTE compared to the placebo group ( $4.2 \%$ vs. $10.2 \%$, respectively). [42]. However, the apixaban group had an increased incidence of major bleeding (3.5\% vs. $1.8 \%)$ and clinically relevant non-major bleeding (7.3\% vs. $5.5 \%)$ compared to the placebo group [42]. Otherwise, there was no difference in overall survival (OS) between these groups [42].

The CASSINI trial has examined the safety and efficacy of rivaroxaban (10 mg daily) in the prevention of cancer-related VTE [43].

Contrary to the AVERT (in which patients were not screened for VTE at the study screening period), participants in the CASSINI underwent venous duplex ultrasound screening for VTE in both legs, prior to entering the trial, and then, every two months, during the entire trial. Patients in whom an occult VTE was diagnosed were excluded from the study [43].

Moreover, CASSINI had a greater proportion of pancreatic cancer participants than AVERT (32\% vs. $13 \%$, respectively) and AVERT had slightly more patients with Khorana scores of 4 or greater than CASSINI (8.9\% vs. 6.6\%) [43].

In CASSINI, the ITT analysis found no significant reduction in VTE events in the rivaroxaban arm compared to placebo after 180 days and no increased risk of major bleeding [43]. However, in the on-treatment analysis, rivaroxaban significantly reduced VTE compared to placebo (2.6\% vs. $6.4 \%)$. These findings suggest that in AVERT and CASSINI studies, the application of the Khorana risk score (e.g., of 2 or above), resulted in a more precise evaluation of low-dose DOAC vs. LMWH therapy, in comparison to the unselected population, assessed in the prior LMWH studies [44].

Implications for the use of primary thromboprophylaxis or extended anticoagulation with DOACs in patients which cancer-related VTE

Balancing the scales of thrombotic and bleeding risks, in the oncology patient population presents unique challenges [45] Although the various cancer-related VTE risk stratification scores exist, the present and future bleeding risk must be considered, such as risks associated with RT, CHT-induced thrombocytopenia, and several other concerns. In fact, large observational or retrospective cohort studies have shown an elevated risk of thrombosis in patients with ALK and ROS1 rearranged non-small-cell lung cancer (NSCLC) compared to those without such rearrangements. Therefore, clinicians should consider a lower threshold to apply thromboprophylaxis in patients with NSCLC and ALK or ROS1 rearrangements, who otherwise have traditional VTE risk scores in the intermediate risk range [45]. Similarly, in the case of molecular aberrations in other tumors, the knowledge that a patient may be at increased thrombotic risk due to the underlying tumor genotype is another valid piece of information that the clinician should consider when determining if a patient can possibly benefit from thromboprophylaxis [45]. In the future, studies on incorporating tumor molecular aberrations into traditional risk scores may enhance the ability of risk scores to identify the patients, who would most likely take advantage of the primary thromboprophylaxis.

Furthermore, there is a scarcity of research exploring the efficacy and safety of extending anticoagulation for cancer-associated VTE beyond the first six months. Current guidelines usually recommend continuing therapy, if the malignancy is still present, or if the patient is still receiving anticancer treatment $[19,46]$. The strongest evidence for treatment of cancer-related VTE with DOACs, beyond the initial six-months, has been derived from the Hokusai VTE Cancer trial, in which the patients were followed for up to one year [47]. The results of this trial support extended anticoagulation beyond the initial six-month treatment period [47]. In addition, a recent cohort study has also reported a reduced risk of VTE recurrence rate at one year, for patients treated with rivaroxaban compared to LMWH and VKA (warfarin) [48].

\section{Conclusions}

Thrombosis has serious impact on morbidity and mortality among patients with malignancies. Cancer-related VTE can be a result of heparanase secretion from the neoplastic tumors (causing degradation of endogenous heparin), abnormal pressure of the tumor mass on blood vessel walls, and adverse effects of different systemic antineoplastic medications, as well as comorbidities, elderly age, or poor performance status of the patient.

Thrombosis is a negative prognostic factor in many patients with cancer, who usually have a greater risk of VTE and bleeding. Early-onset of VTE, especially at the beginning of CHT is a poor prognostic factor for patients with metastatic cancer of the pancreas. Notably, among patients with cancer and acute VTE or PE, LMWH is more effective than an oral VKA for reducing the risk of recurrent VTE, without augmenting the risk of bleeding.

According to the updated ASCO clinical guidelines for the management of VTE in patients with cancer, the direct oral anticoagulants (DOACs), such as apixaban, edoxaban, and rivaroxaban represent a new opportunity for both the prevention and treatment of VTE in this population.

It should be underscored that each time, the application of thromboprophylaxis in a clinical setting should be done according to the dynamic proportions between the benefit of VTE reduction and the risk of bleeding. Further studies are necessary to elucidate the role of tumor genetic abnormalities in the VTE risk, across a wide spectrum of cancers. Hopefully, this may help refine the future risk stratification tools, which (in addition to the patient clinical context and preferences) should enable a more personalized selection of oncology patients for optimal VTE prevention and therapy.

\section{Ethics approval and consent to participate}

Not applicable.

\section{Funding}

Not applicable.

\section{Author contributions}


KR contributes to the all of this paper.

\section{Competing interests}

All authors disclose no competing interests.

\section{References}

1. Timp JF, Braekkan SK, Versteeg HH, Cannegieter SC. Epidemiology of cancer-associated venous thrombosis. Blood 2013; 122: 1712-1723.

2. Lyman GH, Khorana AA, Kuderer NM, et al. Venous thromboembolism prophylaxis and treatment in patients with cancer: American Society of Clinical Oncology clinical practice guideline update. J. Clin. Oncol 2013; 31: 2189-2204.

3. Nasser NJ, Fox J, Agbarya A. Potential Mechanisms of CancerRelated Hypercoagulability. Cancers 2020; 12: 566.

4. Angelini DE, Radivoyevitch T, McCrae KR, Khorana AA. Bleeding incidence and risk factors among cancer patients treated with anticoagulation. Am. J. Hematol 2019; 94: 780-785.

5. Khorana, A.A.; Francis, C.W. Risk prediction of cancer-associated thrombosis: Appraising the first decade and developing the future. Thromb. Res 2018; 164 (Suppl. S1): S70-S76.

6. Ay C, Dunkler D, Marosi C, et al. Prediction of venous thromboembolism in cancer patients. Blood 2010; 116: 5377-5382.

7. Verso M, Agnelli G, Barni S, et al. A modified Khorana risk assessment score for venous thromboembolism in cancer patients receiving chemotherapy: The Protecht score. Intern. Emerg. Med 2012; 7: 291-292.

8. Dickson BC. Venous thrombosis: On the history of Virchow's triad. Univ. Tor. Med. J 2004; 81: 166-171.

9. Nasser NJ, Na'amad M, Weinberg I, Gabizon AA. Pharmacokinetics of low molecular weight heparin in patients with malignant tumors. Anti-Cancer Drugs 2015; 26: 106-111.

10. Vlodavsky I, Gross-Cohen M, Weissmann M, et al. Opposing Functions of Heparanase-1 and Heparanase-2 in Cancer Progression. Trends Biochem. Sci 2018; 43: 18-31.

11. Koliopanos A, Friess H, Kleeff J, et al. Heparanase expression in primary and metastatic pancreatic cancer. Cancer Res 2001; 61 4655-4659.

12. Cohen E, Doweck I, Naroditsky I, et al. Heparanase is overexpressed in lung cancer and correlates inversely with patient survival. Cancer 2008; 113: 1004-1011.

13. Li HL, Gu J, Wu JJ, et al. Heparanase mRNA and Protein Expression Correlates with Clinicopathologic Features of Gastric Cancer Patients: A Meta- analysis. Asian Pac. J. Cancer Prev 2015;16:86538658.

14. Chiu J-J, Chien S. Effects of disturbed flow on vascular endothelium: Pathophysiological basis and clinical perspectives. Physiol. Rev 2011; 91: 327-387.

15. Rak J, Milsom C, Yu J. Tissue factor in cancer. Curr. Opin. Hematol 2008; 15: 522-528.

16. Abbonante V, Chitalia V, Rosti V, et al. Upregulation of lysyl oxidase and adhesion to collagen of human megakaryocytes and platelets in primary myelofibrosis. Blood 2017; 130: 829-831.

17. Nadir Y, Brenner B, Zetser A, et al. Heparanase induces tissue factor expression in vascular endothelial and cancer cells. J. Thromb. Haemost 2006; 4: 2443-2451.

18. Ay C, Pabinger I, Cohen AT. Cancer-associated venous thromboembolism: burden, mechanisms, and management. Thromb Haemost 2017; 117: 219-230.

19. Key NS, Khorana AA, Kuderer NM, et al. Venous thromboembolism prophylaxis and treatment in patients with cancer: ASCO clinical practice guideline update. J Clin Oncol 2019: JCO1901461. https:// ascopubs.org/doi/full/10.1200/JCO.19.01461. Accessed December 13, 2020.

20. Khorana AA, Francis CW, Culakova E, et al. Frequency, risk factors, and trends for venous thromboembolism among hospitalized cancer patients. Cancer 2007; 110(10): 2339-2346.

21. Blom JW, Doggen CJ, Osanto S, Rosendaal FR. Malignancies, prothrombotic mutations, and the risk of venous thrombosis. JAMA 2005; 293(6): 715-722.

22. Khorana AA, Kamphuisen PW, Meyer G, et al. Tissue factor as a predictor of recurrent venous thromboembolism in malignancy: biomarker analyses of the CATCH trial. J Clin Oncol 2017; 35(10): 1078-1085.

23. Ay C, Simanek R, Vormittag R, et al. High plasma levels of soluble P-selectin are predictive of venous thromboembolism in cancer patients: results from the Vienna Cancer and Thrombosis Study (CATS). Blood 2008; 112(7): 2703-2708.

24. Khorana AA, Rao MV. Approaches to risk-stratifying cancer patients for venous thromboembolism. Thromb Res 2007; 120 Suppl 2: S41-S50.

25. Agnelli G, George DJ, Kakkar AK, et al. Semuloparin for thromboprophylaxis in patients receiving chemotherapy for cancer. N. Engl. J. Med 2012; 366: 601-609.

26. Khorana AA, Francis CW, Kuderer NM, et al. Dalteparin thromboprophylaxis in cancer patients at high risk for venous thromboembolism: A randomized trial. Thromb. Res 2017; 151: 8995.

27. Farge D, Bournet B, Conroy T, et al. Primary Thromboprophylaxis in Pancreatic Cancer Patients: Why Clinical Practice Guidelines Should Be Implemented. Cancers 2020; 12: 618.

28. White C, Noble SIR, Watson M, et al. Prevalence, symptom burden, and natural history of deep vein thrombosis in people with advanced cancer in specialist palliative care units (HIDDen): A prospective longitudinal observational study. Lancet Haematol 2019; 6: e79-e88.

29. Chaichana KL, Pendleton C, Jackson C, et al. Deep venous thrombosis and pulmonary embolisms in adult patients undergoing craniotomy for brain tumors. Neurol. Res 2013; 35: 206-211.

30. Osaki T, Saito H, Fukumoto Y, et al. Risk and incidence of perioperative deep vein thrombosis in patients undergoing gastric cancer surgery. Surg. Today 2018; 48: 525-533.

31. Khorana A.A, Dalal M, Lin J, Connolly GC. Incidence and predictors of venous thromboembolism (VTE) among ambulatory high-risk cancer patients undergoing chemotherapy in the United States. Cancer 2013; 119: 648-655.

32. Seng S, Liu Z, Chiu SK, et al. Risk of venous thromboembolism in patients with cancer treated with Cisplatin: A systematic review and meta-analysis. J. Clin. Oncol 2012; 30: 4416-4426.

33. Starling N, Rao S, Cunningham D, et al. Thromboembolism in patients with advanced gastroesophageal cancer treated with anthracycline, platinum, and fluoropyrimidine combination chemotherapy: A report from the UK National Cancer Research Institute Upper Gastrointestinal Clinical Studies Group. J. Clin. Oncol 2009; 27: 3786-3793.

34. Moore RA, Adel N, Riedel E, et al. High incidence of thromboembolic events in patients treated with cisplatin-based chemotherapy: A large retrospective analysis. J. Clin. Oncol 2011; 29: 3466-3473.

35. Fisher B, Costantino J, Redmond C, et al. A Randomized Clinical Trial Evaluating Tamoxifen in the Treatment of Patients with NodeNegative Breast Cancer Who Have Estrogen-Receptor-Positive Tumors. N. Engl. J. Med 1989; 320: 479-484.

36. Fisher B, Dignam J, Wolmark N, et al. Tamoxifen and chemotherapy for lymph node-negative, estrogen receptor-positive breast cancer. J. Natl. Cancer Inst 1997; 89: 1673-1682.

37. Shiroyama T, Hayama M, Satoh S, et al. Successful retreatment with osimertinib after osimertinib-induced acute pulmonary embolism in a patient with lung adenocarcinoma: A case report. Respir. Med. Case Rep 2016; 20: 25-27.

38. Nishio M, Horai T, Horiike A, et al. Phase 1 study of lenvatinib combined with carboplatin and paclitaxel in patients with non-small- 
cell lung cancer. Br. J. Cancer 2013; 109: 538-544.

39. Cabanillas ME, Schlumberger M, Jarzab B, et al. A phase 2 trial of lenvatinib (E7080) in advanced, progressive, radioiodine-refractory, differentiated thyroid cancer: A clinical outcomes and biomarker assessment. Cancer 2015; 121: 2749-2756.

40. Hurwitz H, Fehrenbacher L, Novotny W, et al. Bevacizumab plus Irinotecan, Fluorouracil, and Leucovorin for Metastatic Colorectal Cancer. N. Engl. J. Med 2004; 350: 2335-2342.

41. Ranpura V, Hapani S, Chuang J, Wu S. Risk of cardiac ischemia and arterial thromboembolic events with the angiogenesis inhibitor bevacizumab in cancer patients: A meta-analysis of randomized controlled trials. Acta Oncol 2010; 49: 287-297.

42. Carrier M, Abou-Nassar K, Mallick R, et al. Apixaban to Prevent Venous Thromboembolism in Patients with Cancer. N. Engl. J. Med 2019; 380: 711-719.

43. Khorana AA, So GA, Kakkar AK, et al. Rivaroxaban for Thromboprophylaxis in High-Risk Ambulatory Patients with Cancer. N. Engl. J. Med 2019; 380: 720-728.

44. Agnelli G. Direct Oral Anticoagulants for Thromboprophylaxis in Ambulatory Patients with Cancer. N. Engl. J. Med 2019; 380: 781783.

45. Al-Samkari H, Connors JM. Managing the competing risks of thrombosis, bleeding, and anticoagulation in patients with malignancy. Blood Adv 2019; 3: 3770-3779.

46. Streiff MB, Holmstrom B, Angelini D, et al. NCCN guidelines insights: cancer-associated venous thromboembolic disease, version 2.2018. J Natl Compr Cancer Netw 2018; 16(11): 1289-1303.

47. Raskob GE, van Es N, Verhamme P, et al; Hokusai VTE Cancer Investigators. Edoxaban for the treatment of cancer-associated venous thromboembolism. N Engl J Med 2018; 378: 615-624.

48. Streiff MB, Milentijevic D, McCrae K, et al. Effectiveness and safety of anticoagulants for the treatment of venous thromboembolism in patients with cancer. Am J Hematol 2018; 93(5): 664-671. 